\title{
Designing Structured Design Thinking Framework for Societal System Design in the Unknown Context
}

\author{
Yoshikazu Tomita ${ }^{1} \&$ Takashi Maeno $^{1}$ \\ ${ }^{1}$ Graduate School of System Design andManagement, Keio University, Yokohama, Japan \\ Correspondence: Yoshikazu Tomita, Graduate School of System Design andManagement, Keio University, 4-1-1 \\ Kohhoku Hiyoshi Yokohama, Kanagawa, Japan. Tel: 81-80-3722-1972.
}

Received: July 1, 2018

doi:10.5430/jms.v9n3p103
Accepted: July 26, 2018

Online Published: August 7, 2018

URL: https://doi.org/10.5430/jms.v9n3p103

\begin{abstract}
Currently where everything has increased in complexity, systems designers such as a business model designer are challenged to solve ill-defined problems by creating innovations and designing societal systems. For handling such problems, design thinking has attracted attention as a methodology for solving "ill-defined" problems. However, design thinking cannot create innovations or design societal systems by itself because design thinking cannot guarantee reproducibility in a system design.

In fact, design thinking is effective when applied to the systems approach process and when embedded in its processes. This paper proposes using the advantages of both design thinking and the systems approach to build a structured design thinking framework. This framework integrates the nonstructured design thinking process and the structured systems approach process. We used this framework to redesign a local community in Japan and to design a new concept of an aquarium. We further confirmed that this framework is effective.
\end{abstract}

Keywords: innovation, societal system, system approach, design thinking

\section{Introduction}

Recently, innovation and societal systems have increasingly focused on the discussion of systems. This is particularly true in the area of systems approach (INCOSE, 2014). Innovation and societal systems have similar levels of complexity. Therefore, their designs must incorporate the knowledge of the systems approach. Innovation has many definitions. In management, it is broadly defined as "a process of turning opportunity into new ideas and putting these into widely used practice" (Tidd et al., 2013). The systems approach process and the innovation creation process share common characteristics in many respects, and have a high level of affinity (Scheurer et al., 2012). In both, a successful system development requires finding opportunities in an enormous amount of information, turning such opportunities into ideas, and subsequently using them widely in practice without being bound by any domain. A "societal system" refers to perceiving an entire society as a system with various viewpoints. It is a comprehensive integration of micro and macro phenomena, including economics, organizations, values, ecosystems, and social groups (Luhmann, 1995) (Parsons, 1971). The systems approach is now being actively applied when designing societal systems, especially in projects in the transportation and infrastructure domains (INCOSE, 2014). Currently, systems designers such as a business model designer, must be capable of handling an enormous amount of information obtained from multiple systems and understanding countless stakeholder demands to design sustainable societies. This implies that the systems approach targets are shifting from systems with clear purposes to those with unclear purposes.

In the real world, the "smart grid" and "Industry 4.0" are prominent examples of the societal system. SGAM and RAMI 4.0 are effective reference models for understanding such societal systems. Those models are exemplary in showing how to approach the issue of large-scale and complex societal systems in a structured manner. By defining (limiting) the design purpose, they enable the construction of effective reference models for understanding complex societal systems on a large scale (CEN et al., 2011) (Adolphs, 2015).

How are innovations and societal systems designed? When systems designer begin working on projects targeting on innovation and societal systems, the stakeholders and their concerns are initially unclear. Even the purposes of such systems are often unclear. There is no clear problem definition, no specific methodology to employ, and no single right answer offered as a solution. This is because innovations and societal systems have numerous and extensive variables, 
and different combinations of the variables will result in different future scenarios. Countless possible combinations are available; therefore, from the outset, a clear goal for the system could be not visualized. In conventional systems approach, this is identified as an ill-defined problem, one that should be avoided in the conventional paradigm. When designing a system that has an ill-defined problem, one typically gradually grasps the situation, understands the problem, and makes judgments while one proceeds with the design. In other words, the design must proceed while considering "unknown contexts," as shown in Rumsfeld's ignorance management framework (Israilidis et al., 2013).

How does one resolve "unknown contexts"? One powerful answer is the application of design thinking. It is thought that design thinking is effective in inducing responses to "unknown contexts." Design thinking is a general term for an integrated thinking and performing tasks using a human-centered approach while exploiting diversity. It is effectively employed in an iterative exploration of a vast and unstructured solution space seeking a creative solution (Kelley et al., 2013). GE and IBM are among many corporations that are attempting to incorporate design thinking to generate solutions for creating new values (Kelley et al., 2013).

However, design thinking has its drawbacks. The process of design thinking and its output will not result in solutions immediately. Further, the design process has been discussed and presented by various practitioners, rendering the shared understanding difficult and the design outputs dependent on individuals and hardly reproduced (Braian, 2015).

To overcome these drawbacks, this paper proposes the application of the structured design thinking approach in concept design targeted at innovation and societal systems. This approach is developed to operate effectively in the concept design of societal system, where people's behaviors significantly impact its quality.

This paper has two purposes. The first purpose is to develop a structured design thinking approach that allows for leveraging the power of design thinking in the conceptual design of societal system such as innovation and societal systems. The second purpose is to develop a "structured design thinking framework" to utilize this approach effectively.

\section{Problem}

When products and services alone demonstrate values, the logical research of known markets is effective. However, our society is becoming more interconnected, and the overall picture of a society is changing into a picture of large-scale and complex situation. The meaning of innovation is shifting from simply putting services or products into widely used practices to "open innovation," in which new value is created systematically by connecting people, corporations, and society (Chesbrough, 2006). Societal systems are also changing. The interrelationships between system elements are becoming more complex and expansive. These new relationships and connections will eventually result in unprecedented societal value creation.

Changes occur more dynamically, and the future becomes more uncertain. The interconnections between multiple components will result in new value creation. Further, in many cases, this new value creation occurs in "unknown contexts."

Knowing the "unknown contexts" requires a thinking-outside-the-box mindset. Therefore, design thinking is considered to work effectively for concept design targeted at innovation and societal systems. The design-thinking mindset enables the exploration of the solution space beyond the conventional space.

Design thinking is effective for generating ideas and concepts outside of the existing exploration space that could not be found in quantitative analyses. Thus, much of the design thinking output is often innovative in nature. This is an important aspect in the creation of new values; however, two issues arise when considering the implementation of design thinking. One is the low reproducibility of thinking processes. The other is the unstructured nature of the output.

In conventional system design, such as an organization design, a system has a clear purpose. However, in "unknown contexts" such as an innovation issue, a system has an unclear purpose. In such a system design, the components cannot be categorized into solutions; consequently, it is not possible to define how it can be structured. In other words, no method has been developed yet to structure knowledge in "unknown contexts." This illustrates the exact problem in handling the "ill-defined problem" in our current situation.

Problems can be categorized into two types: the "well-defined" problem and the "ill-defined" problem. (See Fig. 1). A "well-defined" problem is a problem where (1) the problem is clearly defined, (2) the means to solve the problem may be clearly defined, and (3) the solution is unique. In contrast, an "ill-defined" problem is one where (1) the problem cannot be clearly defined, (2) the means to solve the problem is not clearly defined, and (3) the solution is not unique (Matlin, 1989). In conventional problem-solving practice, it is typical to solve "well-defined" problems. However, this is no longer true, especially in the practice of designs targeted at innovation or societal systems. Here, finding the 
solutions require exploring outside the conventional space. This is what one can expect in solving "ill-defined" problems. When comparing the two, solving "ill-defined" problems involves more creativity (Anderson, 2005; Glass et al., 1988). Hence, one must challenge oneself to solve "ill-defined" problems.

\begin{tabular}{|c|c|}
\hline $\begin{array}{c}\text { Well-Defined Problem } \\
\text { Problem that has a unique problem definition }\end{array}$ & $\begin{array}{c}\text { III-Defined Problem } \\
\text { Problem that DOES NOT have a unique problem } \\
\text { definition }\end{array}$ \\
\hline $\begin{array}{c}\text { Well-Structured Problem } \\
\text { Problem that can identify the problem-solving means }\end{array}$ & $\begin{array}{c}\text { Ill-Structured Problem } \\
\text { Problem that CANNOT identify the problem-solving } \\
\text { means }\end{array}$ \\
\hline Well-Posed Problem & $\begin{array}{c}\text { III-Posed Problem } \\
\text { Problem that has a unique solution }\end{array}$ \\
\hline
\end{tabular}

Figure 1. Comparison between well-defined problems and ill-defined problems

However, this presents a dilemma. Even though design thinking is effective in solving "ill-defined" problems, its output, although innovative, is not structured. It is difficult to define unstructured output in concrete terms.

\section{Approach}

In this section, to eliminate the dilemma created when applying design thinking to solve "ill-defined" problems, we propose a structured design thinking approach. This is a type of design thinking in which thinking processes are moderately guided. It is a combination of two different types of approach: the design approach of design thinking and the systems approach of systems engineering. The proposed "structured design thinking approach" exploits the holistic and systematic nature of the systems approach and the exploratory nature of design thinking. Simultaneously, the two approaches supplement one another to eliminate the drawbacks in each approach. This section first defines the scope of this structured design thinking approach, and subsequently provides a process guideline and elaborates the traceability allowed by this approach, which is critical for this thinking process.

\subsection{Purpose}

We initially set the purpose of a "structured design thinking approach." This purpose guarantees reproducibility when using design thinking in the design of societal systems, including business. Three types of reproducibility exist, as follows (Goodman, 2016):

- Methods reproducibility: Adequate information on the methods and data is provided and the same procedure can be repeated.

- Results reproducibility: Independent experiments are conducted with the same procedure as the original experiment to the extent possible, and the same results are obtained.

- Inferential reproducibility: Qualitatively similar results are derived from independent reproduction experiments or the re-analysis of the original research.

Methods reproducibility and inferential reproducibility are important for solving "ill-defined" problems. This is because both possess the reproducibility necessary to obtain a new solution, and the purpose differs from results of reproducibility. Therefore, this study aims to construct a "structured design thinking approach" that is excellent for methods reproducibility and inferential reproducibility.

\subsection{Scope}

In this section, we define the scope of this "structured design thinking approach." We propose that this approach be utilized especially in the concept of systems approach.

Multiple approaches can be used in problem solving. The approach type is decided after considering the differences in the quality of the problems to be solved. Two typical systems approaches are the hard approach and the soft approach. In the hard approach, a model is treated as a replica of the reality and is used as a design drawing (Von Bertalanffy, 1963). In contrast, in the soft approach, a model is not used as a design drawing, and is instead used as a topic material for the dialog between parties (Checkland \& Scholes, 1990). It is considered that problems that are solvable by the analytical hard approach do not require design thinking (the soft approach). In other words, calculating one plus one does not require design thinking. 
For example, the analytical hard approach may often be effective when designing medical systems in certain regions. The process could involve listing the stakeholders, extracting their concerns, examining system life cycles, conducting requirement analyses, defining system requirements, and subsequently architecting the medical systems. With this whole process in accordance with the systems approach, superior medical systems can be designed. The design result can also be validated.

However, when designing systems for improving the quality of life (QOL) of people in a certain region, simply undertaking a design using an analytical approach is not effective. Each individual has a different set of values, rendering it difficult to extract all their concerns. In such cases, the definition of the term QOL is often unclear and hardly shared among stakeholders, rendering it difficult to define the stakeholder requirements. Even if certain systems are eventually designed, it is almost impossible to conduct the validation and verification processes because the unknowns cannot be traced back. Efforts to avoid this situation would often result in only a local resolution of the problem, not a holistic and systemic resolution.

Designing a system targeting at QOL improvement is relatively compatible with the design thinking approach. When a problem is to be solved using the structured design thinking approach, it is reasonable to assume the problem scope to be "unknown" to exploit the features of design thinking.

\subsection{Methodology}

\subsubsection{Systems Engineering (SE) Methodology as a System Approach}

We employed SE methodology in designing the framework for using design thinking effecitvely. In the following section, we discuss what systems engineering methodology is and how it is effective in this study.

<What is system's engineering?>

According to INCOSE handbook (INCOSE 2010), a book published by the world's biggest council for SE, INCOSE (International Council on Systems Engineering), SE is a set of best practices and it is established to design large-scale complicated systems such as the Apollo project. SE has been developed to make the best use of diversity in the engineering field of study. Its standards have been compiled based on military standards in the United States.

SE is not a tool or technique but a methodology. It is a methodology to think about and solve complicated problems. It can be applied in solving problems in any fields. This methodology enables interdisciplinary systems integration and enables people to design solutions in certain domains even if they are inexperienced in those domains.

$\mathrm{SE}$ is considered to be effective not only for designing mechanical systems but also for societal systems. INCOSE is calling for expansion of its role into societal systems (INCOSE 2014). Societal SE requires not only quantitative viewpoints but also qualitative viewpoints. That is why the SE methodology is effectively applied in designing societal systems such as organizations. What is required in the systems engineering is not simply science but the way of thinking between art and science. That is the difference from Engineering.

$<$ SE for societal systems design >

What is societal system? Checkland first analyzed societal systems in an aspect of SE. He discussed societal systems in two aspects (Checkland \& Scholes, 1990). One is an aspect of an artificial system that is man-made and easily recognized as a system such as automobiles, computer programs, laws and mathematics. The other is an aspect of a human-activity system where activities are conducted by people in the society. In this aspect, societal systems could be described as human activities and their interrelations. In this study, we employed systems approach that views systems as elements and their interrelations in dealing with societal systems (Jackson 2013).

\subsubsection{Participatory Systems Analysis (PSA)}

Another approach we employed in designing the structure design thinking approach is PSA. This is a bottom-up approach for problem solving conducted not by just the stakeholders but together with external experts (Bosch 2013).

No man-made system is designed naturally. People intentionally design them. Systems are designed and managed with purposes in order to achieve specific goals.

People interact with systems. Especially in social systems, those who interact with the systems are not limited to their direct users or operators but they include many other stakeholders. Each stakeholder has one's own mental model for the system and its purpose based on his/her own experience, education, understanding or sense of value (Nguyen 2010). Therefore, it is important to design systems using the PSA methodology that involves many stakeholders of the system.

In this paper, we employ this approach to identify problems and to analyze systems utilizing tools and techniques developed in the field of systems approach. 


\subsection{Key Concept From SE and PSA}

\subsubsection{Guide to Thinking}

In a structured design thinking approach, the concept of "guide to thinking" is important. Thinking processes consider when to structure and when not to structure, as the advantages of each approach are known. Thus, exploring the "unknown contexts" can be conducted effectively. It is noteworthy that what guides the direction and important focuses of thinking are the overall thinking processes and the format of each process's outputs, but not the outputs themselves. Innovative ideas may be generated even if the overall thinking processes and output formats are unguided. However, how they may be embodied in structured solutions would not be understood. In addition, if the output contents themselves are guided, the benefits of design thinking that enable exploration beyond the conventional solution space would not be utilized at all.

\subsubsection{Traceability to Think}

In addition to the "guide to thinking" and exploration of "unknown contexts" in the proposed structure design thinking approach, it is important to ensure the "traceability to think." Design thinking engages in the "outside the box" exploration: beyond the existing thinking frameworks. However, outside-the-box thinking involves unknowns, which are thus unstructured. If ideas are created in an unstructured manner, it is difficult to understand how they were created. When an idea is generated from design thinking processes, the idea is typically "generated from the observation of extreme users" or "generated by voting brainstorming outputs." In either case, the result is traceable only with the activity conducted for the idea generation, but not traceable with the overall thinking processes. However, it is always essential to secure the traceability of the outputs. Any solution could cause unexpected failures. It is important to understand the flow and the cause that generated the outputs such that the traceability is always secured. Generating traceable thinking processes has the following benefits: (1) it renders discussions with diverse stakeholders easy, and (2) it enables the investigation of the cause of a problem.

\section{4. "Approach" to "Framework"}

This section introduces a process framework for applying the structured design thinking approach to a conceptual design in an "unknown context." This framework was developed to be used for generic design processes such as organizations, products, and services. This framework is used for guiding from the design purpose to solution through the entire design process. Here, the information should be well examined and gathered to form structured knowledge. In "unknown situations," it is difficult to logically determine what information should be gathered, how it should be gathered, and the purpose of the knowledge formed. Therefore, the concepts of "guide to thinking" and "traceability to think" are essential.

By applying these concepts, this framework promotes a discussion about what meaning is conferred upon the data when categorizing them into information, and how this information is structured to represent knowledge. Hence, information that is not meaningful can be structured into knowledge. The extent of this thinking framework is not limited to the process that occurs in one direction from purpose to solution. Its influence extends to the entire design process. Design thinking is not a linear propagation of thinking, but an iterative advancement. Thus, when the examination of the available information proceeds in design thinking processes, issues and problem definitions are redefined. This refers to the previous process from data to information with new definitions, and new information is structured from data with new meanings. This is how the iteration process. Hence, a structured design thinking framework is designed and developed as a nonlinear framework that allows for iterative thinking.

\subsection{Design Process as a System Life Cycle}

The process of converting information into conceptual design development knowledge in an "unknown context" can be regarded as a system. To design this process as a system, we refer to the generic life cycle defined in ISO15288. As defined in ISO15288, a generic life cycle is recommended to be tailored according to the object to be designed. Consequently, we defined the design process as a system with five processes.

They are "purpose," "problem definition," "value proposition," "solution concept," and "solution". Each subprocess is defined as follows.

Purpose: The process of selecting issues in an environment in which a society and an organization are placed.

Problem Definition: The process of redefining selected issues as specific problems to be solved.

Value Proposition: The process of considering the value to be provided to stakeholders through solutions.

Solution Concept: The process of generating concepts for providing the value. 
Solution: The process of designing ideas as a system such that the value is integrated.

Figure 2 shows a comparison of the generic life cycle defined in ISO 15288 with the tailored design process.

\begin{tabular}{|c|c|c|c|c|c|}
\hline \multirow{2}{*}{$\begin{array}{c}\text { Generic } \\
\text { Iso 15288:2008 }\end{array}$} & \multirow{2}{*}{ Concept } & \multirow{2}{*}{ Develop } & \multirow{2}{*}{ Production } & Utilization & \multirow{2}{*}{ Retirement } \\
\hline & & & & Support & \\
\hline Tailored & Purpose & $\begin{array}{l}\text { Problem } \\
\text { Definition }\end{array}$ & $\begin{array}{c}\text { Value } \\
\text { Proposition }\end{array}$ & $\begin{array}{l}\text { Solution } \\
\text { Concept }\end{array}$ & Solution \\
\hline
\end{tabular}

Figure 2. Tailored design process as a system life cycle

Each process consists of an input, an activity, an output, and an insight. These processes can be depicted in a conventional input-process-output diagram, which can be extended by adding the insight findings. This extension is aimed at intentionally acquiring insights that constitute new discovery, rather than ordinary outputs from each process.

\subsection{Framework Design}

In this section, we will construct a framework using the processes defined in the previous section. A framework is a set of structured viewpoints for understanding and designing purposes. Adjacent processes are connected to one another with an "enabler" relationship and a "utilizer" relationship framework called an "enabler framework" (Shirasaka, 2010). The thinking process is not necessarily required to proceed in the order of the connections. Each process is independent, and the framework users may start thinking from any process. However, the insight acquired in one process should become an input for the next process and this flow is fixed. Fig. 3 shows a diagram of the overall framework that we designed as a "Structured Design Thinking Framework"

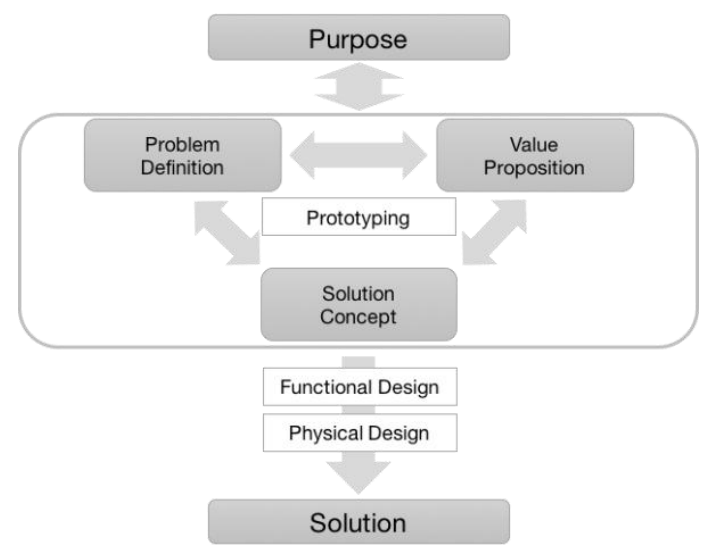

Figure 3. Structured design thinking framework (SDTF)

Each subprocess is defined as an "input," "activity," “output," and "insight."

An explanation of the behavior of the individual constituent elements in each process is as follows:

Input: Each process begins with an input. The input is the known information or outputs from other processes. Because the purpose is to solve "ill-defined problems," the input is not required to be comprehensively organized at this stage.

Activity: In each process, particular activities are conducted in accordance with their purposes based on the inputs to the process. For example, in the problem definition process, such activities include a $2 \times 2$ framework, a journey map, prototyping, and a value graph. A framework user may select the activity to conduct. Here, the mode of performing the selected activity is important, not the activity itself. The user should decide in which mode the activity is conducted, and whether the thinking is being expanded in the divergent mode or being structured in the convergent mode. This is because the output from the same activity is different depending on the mode selected. 
Output: The form of the output differs significantly depending upon (1) the selected activity, and (2) the mode selected by the user of a framework. More importantly, it is visualized regardless of the format type.

Insight: Insight may be discovered at any stage of the thinking process. In many cases, it is discovered when the output is being discussed. When the framework user obtains an insight, the next action is subsequently clarified.

In this framework, the information flows bi-directionally and iteratively.

\section{How to Use the Framework - Two Cases}

In this section, we shall introduce how the proposed "structured design thinking framework" was applied in two cases. The first case was a local community redesign as an example of a societal system, and the second case was a conceptual design for a new aquarium. In each case, the conclusion was confirmed through stakeholder interviews.

\subsection{Case 1: Local Community Redesign}

Summary. This case introduces the application of the proposed framework to a local community redesign in one of the northern islands in Hokkaido, Japan. This outlying island has a population of approximately 2700 and is facing the problems of declining population and declining tax revenue. By identifying the hidden concerns of the people on the island, we concluded that the island is not losing its properties and resources; rather, it is gaining unused properties and resources. Using such tangible and intangible resources, we are constructing a sharing-economy solution.

Stakeholders. Municipal officials, Local business operator(s), University officials, Local residents (including High school student)

Premise. Japan has approximately 1700 municipalities across the country. Each municipality operates multiple systems including medical, educational, traffic, and transportation systems. These systems can be regarded as independent systems constituting to being operated and managed independently in each municipality. However, in reality, they are subsystems of an enormous system centrally operated and managed by the central and local governments. In recent years, owing to the declining birth rate and increasing aging population, it has become difficult for the central government to manage all aspects, from the economy to societal security. The Japanese government has been implementing a policy shift towards a sharing economy to promote self-reliance in municipalities. Hence, a local community redesign based on the features of each municipality is necessary. This will involve redesigning a societal system. This will involve diverse stakeholders and an analytical approach will not be effective. Another issue is the definition of a "sharing economy," which has not been clarified.

Thinking process. Many Japanese municipalities regard the declining population as a negative future scenario and ask the following question: "How can we stop the decline in the population"? However, in this project, the results derived from the issues yielded a completely different problem definition. We have conducted hearings involving various major stakeholders and coordinated the hearing results using this framework. Our problem definition is, "how can we improve people's feelings of well-being along with the decline in the population"?

In societal systems, a shift in population size is regarded as a criterion that can be used to assess the expansion of the economy. However, it is extremely difficult to aim at population increase and the expansion of the economic scale if one considers the reality of this island. Here, a declining population and a shrinking economy should not be the direct targets for resolution. What is required now is to consider the local community and how they can live with such drawbacks. Thus, we have discussed building a sharing economy model where people can feel less dissatisfied with the economic status and more satisfied with their well-being.

In building the sharing economy model, we considered the unused resources from two different perspectives. The first was "waste elimination" and the second was "new value creation." We focused more on the new value creation sharing economy and found the potential in building a "validation island" with the island's resources. By connecting tangible and intangible resources, this island could function as a place for system validation where new societal infrastructures can be tested. Fig. 4 shows the process of this thinking.

Result. We structured the major stakeholders' concerns in the island using the proposed "structure design thinking framework." We conducted hearings and discussions and structured the output by viewing it objectively from a high abstraction level, to avoid being bound by individual concerns and discussions at the low abstraction level. The outputs from the framework are as follows:

- Cost reduction and increased revenue through designing utilization purposes for the unused assets of the island.

- Inspiring and nurturing the will for education to respond to the increase in employment and new occupations on the island. 
- Inspiring "love and pride" of one's home island.

- Developing a new sharing economy model.

By structuring the diverse stakeholders' concerns, we have gained the outputs above by considering the values of the island's assets from multiple viewpoints. The proposed framework is considered to be effective at handling the vast amount of qualitative information and moving towards the goal of value creation. Thus, we consider that the advantage of the framework has been validated.

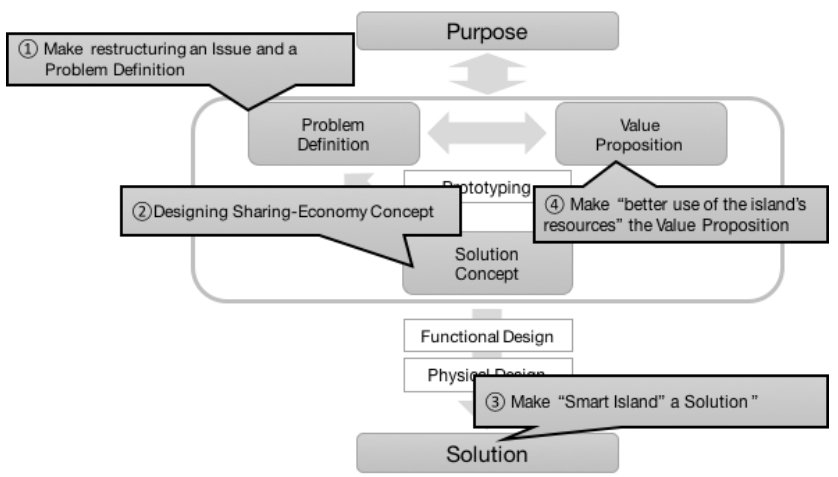

Figure 4. Applying the SDTF to the local community redesign

\subsection{Case 2: New Aquarium Concept Design}

Summary. In Japan, aquariums are becoming commoditized. A major contractor, A, aims at obtaining a top global position among aquarium design and construction companies by conducting a conceptual design of a new aquarium using the "structured design thinking framework." The contractor designed a concept that proposes new purposes and functions for an aquarium.

Stakeholders. Aquarium business operator, Aquarium Designer(s), University officials, System Engineer(s), Assumed customer

Premise. Approximately 100 aquariums exist in Japan, with the number of aquariums per person reportedly being the highest in the world. Aquariums in Japan are categorized into three types. Type 1 refers to aquariums located in coastal areas. Type 2 refers to those in the suburbs, and type 3 are those in urban areas. In recent years, urban aquariums have been evolving into a new type of theme park by merging them with visual entertainment; however, criticism has arose concerning the excessive stage direction that has resulted in aquariums deviating from their original role of promoting wildlife conservation and environmental protection. This role has not been appropriately performed even by traditional aquariums in coastal and suburban areas. It is difficult to admit that they are currently exhibiting anything better or awakening educational interest in audiences. In addition, A is also the constructor of its clients' aquariums. To protect its construction business, it must avoid the situation where its new aquarium jeopardizes the construction of existing aquariums, and the new aquariums must avoid the admission of the existing aquarium visitors. Hence, A had to generate a new aquarium concept.

Thinking process. In a typical aquarium construction, the design process is very tightly controlled and managed to eliminate uncertainties. The target system boundaries are clear and must be managed such that it is possible to analyze the requirements comprehensively. However, in this project, using sensing technologies, a large quantity of data and signals had to be collected inside the aquarium building to obtain new forms of information with new meanings. Using the "structured design thinking framework," this was subsequently structured into new knowledge about the aquarium. Here, we attempted to create "unknown contexts" in the aquariums. This is because differentiation is difficult in under "known" and certain circumstances. It is necessary to incorporate values from completely new domains that are not discussed by other competitors. Hence, particularly during the value proposition process, the extraction of value and the functions to realize it were selectively performed, and the requirements for the information and facilities to realize it were extracted. This was effective for structuring knowledge from the information in "unknown contexts" and for developing a business model to utilize such knowledge as key success factors, aiming at changing the aquarium business. Fig. 5 shows this thinking process. 
Results. Conventionally, the architecture and the business model for aquariums are designed based on a fixed structure, rendering a new value creation difficult. To obtain new design variables, we intentionally created "unknown contexts" by building a structure and subsequently breaking it deliberately. The output from the framework is as follows:

- Discovery of a new visitor segment.

- Construction of a new value creation point.

- $\quad$ Overall design enabling collaboration with existing aquariums.

- The possibility of use as a conceptual design framework for investigating nonaquarium commercial facilities.

Using the framework, we have successfully focused on a specific domain and created "unknown contexts" there without demolishing the whole structure of the aquarium architecture and its business model.

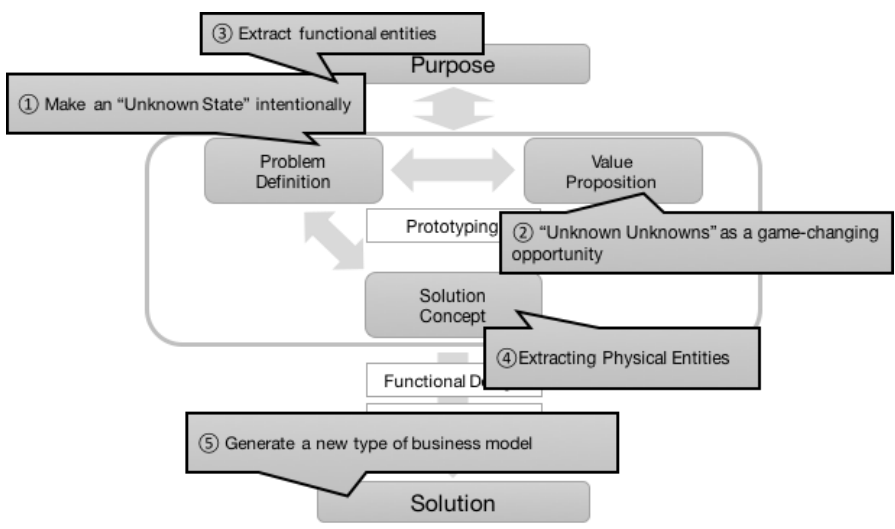

Figure 5. Applying the SDTF to the concept design of the new type of aquarium

\section{Conclusion and Future Work}

Currently, innovation and societal systems are becoming increasingly large-scale and complex. In many cases, such systems are where people's behaviors impact significantly on their quality.

As stakeholders comprising a system become diverse, the values sought by them is becoming varied. Consequently, we are faced with many ill-defined problems in many domains. Each of these problems needs to be urgently solved; however, it is difficult to capture and define the problems. In this paper, we proposed a structured design thinking approach for maximizing the benefits of design thinking during the conceptual design in the societal system design, and introduced a framework for applying this approach. PSA is utilized not only for systems analysis but also for designing systems. When utilized for designing, this process is iteratively conducted and constantly gets feedbacks. PSA is an evolutional process and never ends. Especially societal systems such as two cases need to be evolutional (Bosch 2011). Evolutional systems are constantly evaluated and verified. They are not evaluated and verified when the design is finished but they are evaluated and verified while they are designed. This means that this frame work was evaluated and verified by stakeholders throughout design processes.

This structured design thinking approach complements the hard and soft approaches, which are typical approaches for problem solving. It is an approach that maximizes the benefits of both the hard approach (targets are addressed through modeling) and the soft approach (targets are addressed as a whole.) By applying the concepts of "guide to thinking" and "traceability to think" to design thinking, we realized this complementary approach.

Thus, the "Structuree Design Thinking Framework" for applying this "structured design thinking approach" incorporates the processes - purpose, problem definition, value proposition, solution concept, and solution - and is characterized by an activity flow. The whole framework is structured and designed to generate iterative thinking.

This approach and framework contribute to improving the reproducibility of thinking processes that occur when solving problems using design thinking, and the utility of the output. We explained the efficacy and promise of these contributions using the cases of a local community redesign and a new aquarium concept design.

Based on the results of this study, we have clarified the future work to further advance this study. Although this framework results in an overall picture of thinking processes, it does not reveal the process to start with and the 
activities to choose. These are relatively dependent on the experiences of each individual framework user, as are the finding insights. The method to find the important insights and how to make them inputs to the next activity are also dependent on the experiences of each individual. We believe that these must be defined and clearly guided. In addition, because the efficacy of this approach and framework were verified by limited cases only, we must pursue further verifications using other cases and extract further improvements.

Although many areas of improvement exist, we believe that the results of this study represent an important opportunity to design a society as a system with increasing complexity. In a world with an ever-increasing number of components to be assessed, very large amounts of data must be collected, analyzed, and visualized. More importantly, solutions to problems must be obtained beyond the conventional concepts. Under such circumstances, it is easily assumed that artificial intelligence will be critical in generating solutions to many different problems. With such technological progress, people should not only analyze what is "known," but also explore the world of "unknowns." We expect that the results of this study will facilitate in the allocation and promotion of roles and activities to be played by people.

\section{References}

Adolphs, P., et al.. (2015). Reference architecture model industrie 4.0 (rami4. 0). ZVEI and VDI, Status Report.

Anderson, J. R. (2005). Cognitive psychology and its implications. Macmillan.

Bosch, O. J. H., \& Nguyen, N. C. (2011). Establishing the Global Learning Laboratories NET for managing complex problems. Working Paper, Brisbane, Australia, School of Integrative Systems, the University of Queensland.

Bosch, O., et al.. (2013). Addressing the Critical Need for "New Ways of Thinking" in Managing Complex Issues in a Socially Responsible Way, Business System Review. The 1st B.S.Lab International Symposium, Special Issue, 2(2), 48-70.

Braian, L. (2015). Implementing design thinking 1: focus on the outcome not the process. Design Sojourn. Retrieved from http://designsojourn.com/implementing-design-thinking-1-focus-on-the-outcome-not-the-process/

CEN, CENELEC, \& ETSI. (2011). Smart Grid Reference Architecture. CEN-CENELEC-ETSI Smart Grid Coordination Group.

Chaffey, C., \& Wood, D. (2005). Business information management: improving performance using information systems. Prentice Hall, UK.

Checkland, P., \& Scholes, J. (1990). Soft systems in action. John Wiley and Sons, Chichester, UK.

Chesbrough, H. W. (2006). Open innovation: The new imperative for creating and profiting from technology. Harvard Business Press.

Glass, A. L., \& Holyoak, K. J. (1988). Cognition (2nd ed.). Random House, New York, US.

Goodman, S. N., Fanelli, D., \& Ioannidis, J. P. (2016). What does research reproducibility mean?. Science Translational Medicine, 8(341), 341ps12.

Institute of Electrical and Electronics Engineers (IEEE). (2005). IEEE Std 1220TM-2005, IEEE Standard for Application and Management of the Systems Engineering Process. New York, US.

International Council on System Engineering (INCOSE). (2010). INCOSE System Engineering Handbook, Version 3.2 .

International Council on System Engineering (INCOSE). (2014). A World in Motion: Systems Engineering, Vision 2025, INCOSE. San Diego, US.

Israilidis, J., et al.. (2013). Ignorance management. Management Dynamics in the Knowledge Economy.

Jackson, M. (2003). Systems Thinking: Creative Holism for Managers. John Wiley \& Sons, Inc.

Kelley, T., \& Kelley, D. (2013). Creative confidence: Unleashing the creative potential within us all. Crown Business.

Kolko, J. (2015). Design thinking comes of age. Harvard Business Review, 93(9).

Luhmann, N. (1995). Social systems. Stanford University Press, (CA, US).

Matlin, M. W. (1989). Cognition. New York: Holt, Rinehart and Winston.

Nguyen, N.C., et al.. (2010). Creating 'Learning Laboratories' for Sustainable Development in Biospheres: A Systems Thinking Approach. Systems Research and Behavioral Science, Syst. Res, 28, 51-62.

Parsons, T. (1971). The system of modern societies. Prentice Hall, New Jersey, US. 
Plattner, H. (2010). An introduction to design thinking process guide. The Institute of Design at Stanford, Stanford University, CA, US.

Rowley, J. E. (2007). The wisdom hierarchy: representations of the DIKW hierarchy. Journal of Information Science.

Scheurer, R., \& Strosnider, D. (2012). Maximizing innovation with systems engineering. Conference Presentation, National Defense Industrial Conference.

Shafaat, A., \& Kenley, R., C. (2015). Exploring the role of design in systems engineering. 25th Annual INCOSE International Symposium, Seattle, US.

Shirasaka, S. (2010). A standard approach to find out multiple view points to describe an architecture of social systemsdesigning better payment architecture to solve claim-payment failures of Japan's insurance companies. 21st Annual INCOSE International Symposium.

Tidd, J., \& Bessant, J. R. (2013). Managing Innovation: Integrating Technological, Market and Organizational Change (5th ed.).

Von Bertalanffy, L., \& Rapoport, A. (Eds.). (1963). General systems. Society for general systems research. 